CLINICAL STUDY

\title{
A questionnaire survey on the management of Graves' orbitopathy in Europe
}

The European Group of Graves' Orbitopathy: P Perros, L Baldeschi ${ }^{1}$, K Boboridis ${ }^{4}$, A J Dickinson ${ }^{5}$, A Hullo ${ }^{6}$, G J Kahaly 8 , P Kendall-Taylor ${ }^{9}$, G E Krassas ${ }^{10}$, C M Lane ${ }^{11}$, J H Lazarus ${ }^{12}$, C Marcocci ${ }^{13}$, M Marino ${ }^{13}$, M P Mourits ${ }^{2}$,

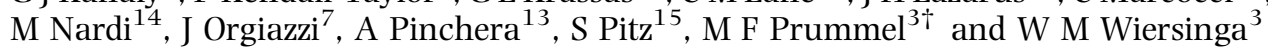

Department of Endocrinology, Freeman Hospital, Newcastle upon Tyne, UK, ${ }^{1}$ Department of Ophthalmology, ${ }^{2}$ Orbital Center, Department of Ophthalmology, and ${ }^{3}$ Department of Endocrinology, Academic Medical Center, Amsterdam, The Netherlands, ${ }^{4}$ University Department of Ophthalmology, Ahepa Hospital, Thessaloniki, Greece, ${ }^{5}$ Department of Ophthalmology, Royal Victoria Infirmary, Newcastle upon Tyne, UK, Services of ${ }^{6}$ Ophthalmology and ${ }^{7}$ Endocrinology, Centre Hospitalier Lyon-Sud, Lyon, France, ${ }^{8}$ Department of Medicine I, Gutenberg University Hospital, Mainz, Germany, ${ }^{9}$ Medical School, University of Newcastle upon Tyne, Newcastle upon Tyne, UK, ${ }^{10}$ Department of Endocrinology, Panagia General Hospital, Thessaloniki, Greece, ${ }^{11}$ Cardiff Eye Unit, University Hospital of Wales, Heath Park, Cardiff, UK, ${ }^{12}$ School of Medicine, Cardiff University, Llandough Hospital, Cardiff, UK, Departments of ${ }^{13}$ Endocrinology and ${ }^{14}$ Neuroscience Section of Ophthalmology, University of Pisa, Pisa, Italy and ${ }^{15}$ Department of Ophthalmology, Johannes Gutenberg-University, Mainz, Germany

(Correspondence should be addressed to P Perros; Email: petros.perros@ncl.ac.uk)

$\dagger$ Deceased.

\begin{abstract}
Objective: To determine management patterns among clinicians who treat patients with Graves' orbitopathy (GO) in Europe.

Design and methods: Questionnaire survey including a case scenario of members of professional organisations representing endocrinologists, ophthalmologists and nuclear medicine physicians.

Results: A multidisciplinary approach to manage GO was valued by $96.3 \%$ of responders, although $31.5 \%$ did not participate or refer to a multidisciplinary team and $21.5 \%$ of patients with GO treated by responders were not managed in a multidisciplinary setting. Access to surgery for sight-threatening GO was available only within weeks or months according to $59.5 \%$ of responders. Reluctance to refer urgently to an ophthalmologist was noted by $32.7 \%$ of responders despite the presence of suspected optic neuropathy. The use of steroids was not influenced by the age of the patient, but fewer responders chose to use steroids in a diabetic patient $(72.1$ vs $90.5 \%, P<0.001)$. Development of cushingoid features resulted in a reduction in steroid use $(90.5$ vs $36.5 \%, P<0.001)$ and increase in the use of orbital irradiation (from $23.8 \%$ to $40.4 \%, P<0.05$ ) and surgical decompression (from 20.9 to $52.9 \%$, $P<0.001)$. More ophthalmologists chose surgical decompression for patients with threatened vision due to optic neuropathy, who were intolerant to steroids than other specialists $(70.3$ vs $41.8 \%$, $P<0.01)$.

Conclusion: Deficiencies in the management of patients with GO in Europe were identified by this survey. Further training of clinicians, easier access of patients to specialist multidisciplinary centres and the publication of practice guidelines may help improve the management of this condition in Europe.
\end{abstract}

European Journal of Endocrinology 155 207-211

\section{Introduction}

The management of Graves' orbitopathy (GO) continues to be challenging for both patients and clinicians. Such patients are best managed in a multidisciplinary setting with input by both specialist physicians and ophthalmologists (1). A previous European questionnaire survey conducted in 1996 (2) showed a marked variation among thyroid experts across Europe in the treatment of GO, indicative of the lack of well-designed studies in this area. Since the collection of that data, a number of new pieces of evidence in this field has emerged including prospective randomised studies (3-7), studies on the effects of smoking on treatment outcomes, and studies addressing safety issues of medical treatments $(8-11)$.

The aim of this study was to gain insight on how patients with GO are managed in Europe and thus assess: (a) accessibility of patients with GO to treatments for their disease, (b) thresholds for diagnosing optic neuropathy and referral to ophthalmologists and (c) the impact of new evidence on management by comparing responses with the previous questionnaire survey (2). 


\section{Methods}

A questionnaire was drafted and circulated to The European Group on Graves' Orbitopathy (EUGOGO) members. After discussions it was modified to its final form. The questionnaire requested general background information about the organisation of services for patients with GO in the responders' locality and accessibility of these services to patients with GO. It was followed by the description of the index case (Table 1). The questionnaire collected information on the following:

(a) Thresholds for diagnosis, referral to an ophthalmologist and initiation of treatment for optic neuropathy.

(b) The effects of clinical variants (diabetes and younger age) on treatment choices.

(c) The influence of the development of iatrogenic Cushing's syndrome on choice of additional treatment for optic neuropathy.

(d) Choice of treatment for hyperthyroidism in the active and inactive phases of GO.

The questionnaire was sent to three professional European organisations whose members are potentially involved in the management of patients with GO: the European Thyroid Association (ETA), European Society of Ophthalmic Plastic and Reconstructive Surgery (ESOPRS) and the European Association of Nuclear Medicine (EANM).

The questionnaires were sent to members of the ETA by e-mail $(n=333)$ or by post to those with no e-mail addresses $(n=140)$. Six months later, reminders were sent by e-mail or post to those who had not responded. Similar requests by e-mail were sent to members of the EANM and ESOPRS. One hundred and twenty-two responses were received. Of these, 14 were from members who declared that they had no involvement

Table 1 Case scenario: patient with moderate to severe Graves' ophthalmopathy and hyperthyroidism.

\section{Index case}

A 65-year-old female patient presents with typical symptoms of hyperthyroidism. She also gives a 6-week history of uncomfortable watery eyes; lid swelling in the mornings and double vision on upward and lateral gaze. She smokes 15-20 cigarettes per day. On examination, she is found to be moderately thyrotoxic. She has an easily palpable but small symmetrical goitre. There is marked periorbital oedema, redness of the conjunctivae, bilateral chemosis and obvious restriction of eye movements on upward gaze, and attempts to look up provoke retro-orbital pain. Proptosis is $19 \mathrm{~mm}$ bilaterally. Her visual acuity is normal $(6 / 6-1.0$, bilaterally on the Snellen chart). On direct questioning, she admits to being aware that colours appear less bright than they did 2 weeks earlier. Fundoscopy shows normal optic disks. Biochemistry confirms thyrotoxicosis (free thyroxine $52 \mathrm{pmol} / \mathrm{l}$, normal range 11-23; total tri-iodothyronine $9.3 \mathrm{nmol} / \mathrm{l}$, normal range 1-2.9; thyroid-stimulating hormone $<0.05 \mathrm{mU} / \mathrm{l}$ ). with patients with GO and provided no data for the analysis. These responders were excluded from further analysis. Thus, there was a total of 108 valid responders of whom 55 were ETA, 37 ESOPRS and 16 EANM members. Twenty-one countries were represented. Most responses came from Germany $(n=28)$, UK $(n=19)$, Italy $(n=11)$, Denmark $(n=6)$, France $(n=6)$, Spain $(n=5)$ and Turkey $(n=5)$. Questionnaires were collected and the data analysed using GraphPad Prism (GraphPad Software Inc., San Diego, CA, USA). Chisquared test was used to compare responses.

\section{Results}

\section{Background information}

Multidisciplinary teams (MDTs) for management of GO. The majority of responders $(96.3 \%)$ described a multidisciplinary setting for management of GO as 'valuable'. More than half of all the responders (54.6\%) participated in an MDT for the management of GO. A significant minority $(31.5 \%)$ neither participated nor referred to an MDT and estimated numbers of patients with GO seen by this group of responders (not participating or referring to MDT) in the previous 6 months was 418 out of the total of $1948(21.5 \%)$ patients seen by all responders.

Access of patients with optic neuropathy to specialist orbital surgery. Access to a specialist surgeon (for orbital decompression) was available 'within months' in $13.8 \%$ and 'within weeks' in $45.7 \%$ of centres.

Practice guidelines. The overwhelming majority of responders (92\%) stated that they would welcome the publication of practice guidelines for GO.

\section{The index case}

Diagnosis of optic neuropathy. Given the scenario of active eye disease of recent onset with a history of colour desaturation, but normal visual acuity (Table 1), an urgent referral to an ophthalmologist was judged appropriate by $67.3 \%$ of responders, non-urgent referral by $27.1 \%$ and no referral by $5.6 \%$.

In response to additional clinical information about optic nerve function (reduction of colour vision on Ishihara plates to $12 / 15$ on the right and $14 / 15$ on the left, possible right peripheral field defect and marginally delayed visual evoked potential responses on the right), while visual acuity was preserved (6/6 or 1 on Snellen chart bilaterally), $78 \%$ of the responders felt that the diagnosis of optic neuropathy was very likely or probable and $21.9 \%$ unlikely. 
Additional information implying the presence of optic neuropathy (visual acuity $6 / 12$ or 0.5 on the right and $6 / 9$ or 0.67 on the left and a blurred disk margin on the right on fundoscopy) led to the initiation of treatment by $99.05 \%$ of responders.

Treatment of optic neuropathy. Steroid of some form (oral, i.v. or subconjunctival/retrobulbar) was recommended by the vast majority of responders (90.5\%). i.v. steroids (alone or in combination with other treatments) was the most frequently chosen treatment $(69.5 \%)$, followed by oral steroids alone or in combination with other treatments $(35.2 \%)$, radiotherapy (alone or in combination with other treatments) by $23.8 \%$ of responders and surgical decompression (alone or in combination with other treatments) by $20.9 \%$ of responders (Fig. 1).

A younger age (32 years compared to 65 years) did not influence the choice of treatments (Fig. 1).

The presence of diabetes led to a significant reduction in the use of steroids (from 90.5 to $72.1 \%, P<0.001$ ), a slight (non-significant) increase in the use of orbital irradiation (from 23.8 to $27.9 \%$ ), more use of surgical decompression (from 20.9 to $29.8 \%$ ) and more use of cyclosporine (from 3.8 to $9.6 \%$ ), although these changes did not reach statistical significance (Fig. 1).

Responders were asked whether their treatment choice would alter in the light of marked cushingoid side effects and ongoing threat of optic neuropathy (visual acuity is $6 / 6$ or 1 bilaterally, colour vision on

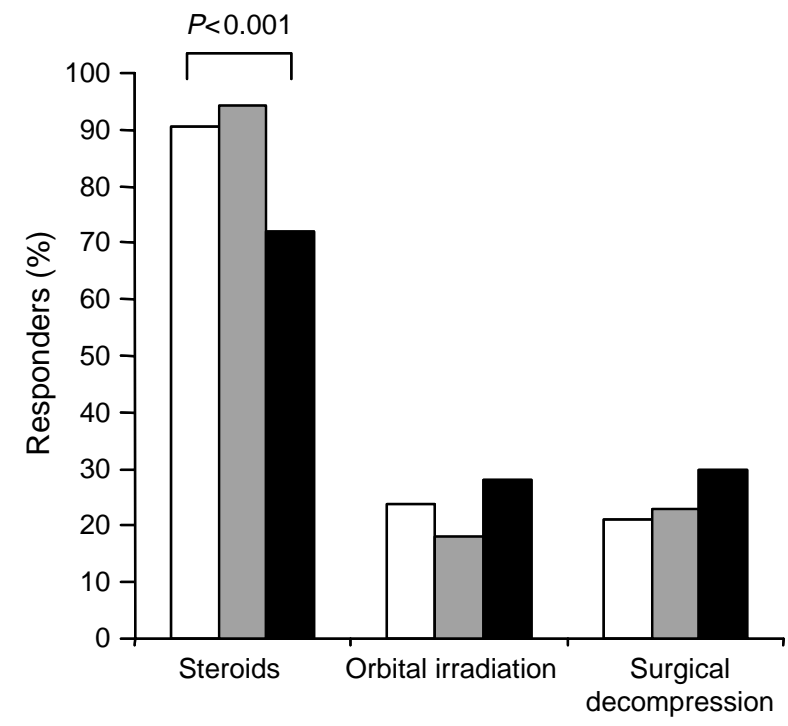

Figure 1 Treatment choices for index case with optic neuropathy at first presentation with variants (diabetes and younger age). Responders were allowed to choose more than one treatment options. Open bars, age 65 years no diabetes; grey bars, age 32 years no diabetes; black bars, age 65 years with diabetes. A significant decline in use of steroids in the presence of diabetes was observed. The diagnosis of diabetes or younger age had no significant influence on other choices.
Ishihara plates $13 / 15$ on the right and $15 / 15$ on the left, a possible relative afferent pupillary defect on the right, normal looking optic discs and the right orbit feeling very 'tight' on ballottement). There was a major shift away from the use of steroids (down from 90.5 to $36.5 \%, P<0.001)$, and a rise in the use of orbital irradiation (from 23.8 to $40.4 \%, P<0.05$ ) and surgical decompression (from 20.9 to $52.9 \%, P<0.001$ ) (Fig. 2). Ophthalmologists were more likely to consider surgical decompression under these circumstances than endocrinologists and nuclear medicine physicians (70.3 vs $41.8 \%, P<0.01)$. A younger age (32 years) led to a slight reduction in the use of orbital irradiation (34 vs $40.4 \%$ ) and a slight increase in the use of steroids (Fig. 2), but neither of these changes was significant. The diagnosis of diabetes resulted in a modest nonsignificant reduction in the use of oral steroids and a rise in the use of other immunosuppressive therapies (azathioprine, cyclosporine, somatostatin analogues) and no change in the use of orbital irradiation (Table 2).

Treatment for hyperthyroidism. The majority of responders $(90.9 \%)$ were in favour of antithyroid drugs (Table 2) as first line therapy. Radioiodine was the least popular option (2\%). Thyroidectomy was advocated by $3 \%$ of responders.

Following restoration of euthyroidism, responders were asked if they would prefer a second line treatment of hyperthyroidism. Four options were offered: radioiodine alone, radioiodine with prophylactic steroids, thyroidectomy and 'other'. There was a major shift in

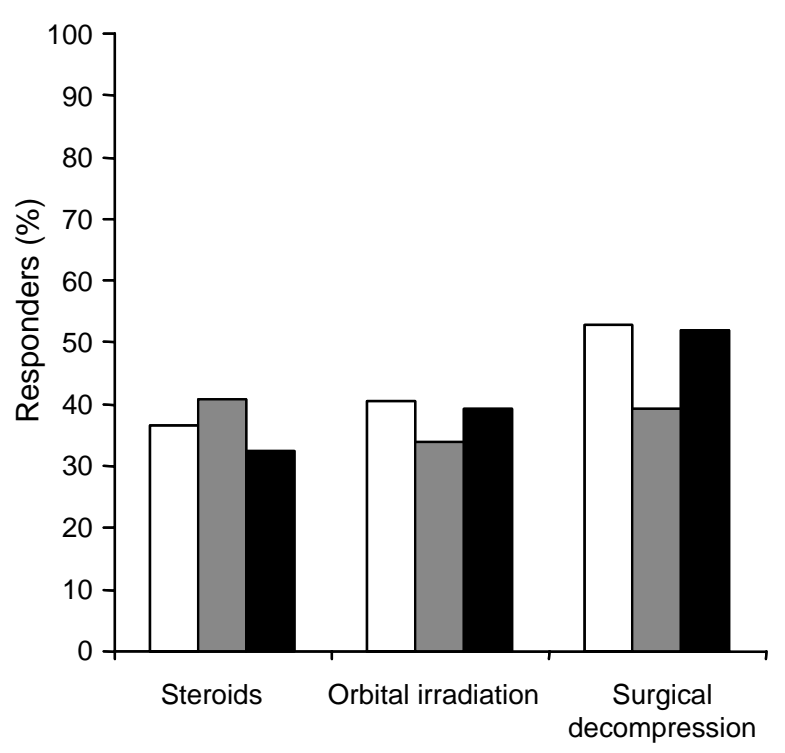

Figure 2 Treatment choices for index case after development of cushingoid features due to steroid treatment. The optic nerves are still threatened but under control while on steroids. Responders were allowed to choose more than one treatment options. Open bars, age 65 years no diabetes; grey bars, age 32 years no diabetes; black bars, age 65 years with diabetes. Younger age or the presence of diabetes had no significant impact on treatment choices. 
Table 2 Choice of antithyroid treatment of index case with hyperthyroidism and Graves' Orbitopathy.

\begin{tabular}{|c|c|c|c|}
\hline & $\begin{array}{l}\text { At presentation (first line } \\
\text { treatment), while patient } \\
\text { has active eye disease }\end{array}$ & $\begin{array}{l}\text { After restoration of euthyroidism } \\
\text { with first line treatment, while } \\
\text { patient has active eye disease }\end{array}$ & $\begin{array}{l}\text { Eight months after presentation } \\
\text { when the patient developed } \\
\text { agranulocytosis on carbimazole } \\
\text { and eye disease is inactive }\end{array}$ \\
\hline Antithyroid drugs & $90.9 \%$ & - & $13.3 \%$ (switch to propylthiouracil) $^{*}$ \\
\hline Radioiodine & $0 \%$ & $2 \%$ & $4.1 \%^{\star \star}$ \\
\hline $\begin{array}{l}\text { Radioiodine with oral } \\
\text { steroid prophylaxis }\end{array}$ & $3 \%$ & $43.6 \%{ }^{*}$ & $43.9 \% *$ \\
\hline Thyroidectomy & $3 \%$ & $34 \% *$ & $34.7 \%$ * \\
\hline Other & $3 \%$ & $20.2 \%$ * & $4.1 \%$ \\
\hline
\end{tabular}

${ }^{*} P<0.001$ compared with left hand column; ${ }^{* *} P<0.05$ compared with left hand column.

favour of the use of radioiodine with steroid prophylaxis (from 3 to $43.6 \%, P<0.001$ ) and thyroidectomy (from 3 to $34 \%, P<0.001$ ) (Table 2 ).

Responders were asked to indicate their preference for treatment of thyrotoxicosis 8 months after the initial presentation when the patient developed agranulocytosis, while the eye disease had burnt out. This had little impact on the use of radioiodine or surgery (Table 2).

\section{Comparisons with previous questionnaire}

Joint clinics were utilised by $40 \%$ of responders in 1996 (2). In the present survey $65.4 \%$ of responders had access to a joint clinic.

Among treatments for severe GO, there was greater use of steroids $(P<0.001)$ and lesser use of radiotherapy $(P<0.001)$ in the present survey than in 1996 . Treatment modalities for hyperthyroidism as first line therapy were not significantly different in the present survey than in 1996 (Table 3).

\section{Discussion}

GO is an autoimmune condition with a complex pathogenesis. Although most cases have mild disease requiring no, or minimal intervention, severe cases can be sight threatening and cause significant psychosocial morbidity. Management of progressive severe cases can be very difficult. Because of the relative rarity of GO, expertise is difficult to accumulate and maintain except in tertiary referral centres, which have an interest in this condition. Furthermore, randomised controlled trials of different therapies have only started to emerge in the last decade and the impact of this relatively new information on clinical practice is unknown. Knowledge of present practice by specialists who manage patients with GO is valuable in identifying deficits in training, education and expertise, and in planning services for patients with this condition. One way of gathering such information is by distribution of questionnaires targeting specialists who are likely to be involved in the management of GO. The response rates to our questionnaire were lower than expected and it is difficult to be certain that the views expressed are representative. However, it is likely that the majority of those who did not reply were either clinically inactive, managed very few patients with GO, or referred them to other specialists, therefore, we believe that the opinions expressed among the responders affect a large number of patients with GO in Europe. This is supported by the estimates of the numbers of patients with GO provided by responders who were seen in the preceding 6 months $(n=1948)$ and the incidence of GO in the population (12). In comparison to the 1996 survey (2), the mean number of patients with GO seen per responder over 6 months was lower (56 patients per responder in 1996,18 patients per responder in the present survey). Interpretation of these data is difficult as the specialities targeted by the two surveys were different.

This survey has identified potential inadequacies of clinical services, particularly orbital surgery available to patients with GO in Europe, as illustrated by the following findings:

(a) Specialist orbital surgery for optic neuropathy (the most dreaded complication of GO) was reported by $59.5 \%$ of responders to be available only within 'weeks' or 'months', a timeframe that might arguably lead to sight loss or use of less efficacious treatments associated with more side effects.

(b) When the index case developed troublesome cushingoid features, while the optic nerves were still threatened, one-third of the responders

Table 3 Comparisons between ETA member responses between 1996 and the present survey. Responders from outside Europe were excluded from the analysis.

\begin{tabular}{lcc}
\hline & $\begin{array}{c}\mathbf{1 9 9 6} \\
\text { survey }\end{array}$ & $\begin{array}{c}\text { Present } \\
\text { survey }\end{array}$ \\
\hline Total number of responders & 91 & 108 \\
Treatment used for severe progressive GO & & \\
Steroids (\%) & $\sim 35$ & $90.9^{*}$ \\
Orbital irradiation (\%) & $\sim 45$ & $21.8^{*}$ \\
Surgery (\%) & $\sim 30$ & 21.8 \\
Other immunosuppressives (\%) & $\sim 20$ & 14.5 \\
Treatment for thyrotoxicosis at presentation & & \\
Antithyroid drugs (\%) & 84 & 90.9 \\
Thyroidectomy (\%) & 10 & 3 \\
Radioiodine (\%) & 4 & 0 \\
Radioiodine with prophylactic steroids (\%) & 2 & 3
\end{tabular}

${ }^{\star} P<0.001$. 
persevered with steroid therapy rather than opting for surgical decompression.

(c) $21.5 \%$ of patients with GO were managed outside a multidisciplinary setting, despite the fact that $96.3 \%$ of the clinicians responding to the questionnaire described multidisciplinary clinics as valuable.

The scenario of the index case described a patient who was highly likely to have early optic nerve compression. It is therefore disturbing that $21.9 \%$ of responders felt that optic neuropathy was unlikely and that a small minority $(5.4 \%)$ elected not to refer the patient to an ophthalmologist or defer referral until the patient became euthyroid. A greater proportion of ophthalmologists thought that optic neuropathy was probable or very likely and suggested an urgent referral than the other two specialities (endocrinology and nuclear medicine), which suggests that more training and education is required on the management of GO. Normal visual acuity is compatible with optic neuropathy (13), yet preservation of normal visual acuity in the case scenario appeared to have deterred responders from making a diagnosis of optic neuropathy even in the context of other compelling clinical features of this complication. These findings are suggestive that specialist training in assessing and interpreting diagnostic tests in patients with GO has been lacking across Europe, and EUGOGO is addressing this by its educational activities.

Evidence-based practice appeared to be implemented as illustrated by the choices of treatments for optic neuropathy being mainly i.v. steroids (6), the rare use of radioiodine as first treatment for thyrotoxicosis (3) compared with the previous survey (2). However, the recommendation to avoid the use of orbital irradiation in patients with diabetes mellitus appears to have been overlooked (11).

The suggestion of practice guidelines was received positively by the overwhelming majority of responders and this is a task that professional organisations leading the field should consider in view of the deficiencies identified above in present practice.

In conclusion, this survey has identified significant deficiencies in the quality of care delivered to patients with GO in Europe and possible deficiencies in the ability to diagnose optic neuropathy, which may reflect suboptimal training of specialists managing GO. Therapeutic decisions appeared to be largely evidence-based. Clinicians who participated in this survey expressed a desire for practice guidelines.

\section{The European Group on Graves' Orbitopathy}

European Group on Graves' Orbitopathy (EUGOGO) is a multidisciplinary consortium of clinicians from European centres who share a commitment to improve the management of Graves' orbitopathy, have a good track record in clinical research in this field, are regional or national referral Centres for the treatment of this disease and manage patients in a multidisciplinary setting with input from endocrinologists and ophthalmologists.

\section{References}

1 Anonymous. Classification of eye changes of Graves' disease. Thyroid 19922 235-236.

2 Weetman AP \& Wiersinga WM. Current management of thyroidassociated ophthalmopathy in Europe. Results of an international survey. Clinical Endocrinology 199849 21-28.

3 Bartalena L, Marcocci C. Bogazzi F, Manetti L, Tanda ML, Dell'Unto E, Bruno-Bossio G, Nardi M, Bartolomei MP, Lepri A, Rossi G, Martino E \& Pinchera A. Relation between therapy for hyperthyroidism and the course of Graves' ophthalmopathy. New England Journal of Medicine $199833873-78$.

4 Mourits MP, van Kempen-Harteveld ML, Garcia MB, Koppeschaar HP, Tick L \& Terwee CB. Radiotherapy for Graves' orbitopathy: randomised placebo-controlled study. Lancet 2000355 1505-1509.

5 Gorman CA, Garrity JA, Fatourechi V, Bahn RS, Petersen IA, Stafford SL, Earle JD, Forbes GS, Kline RW, Bergstralh EJ, Offord KP, Rademacher DM, Stanley NM \& Bartley GB. A prospective, randomized, double-blind, placebo-controlled study of orbital radiotherapy for Graves' ophthalmopathy. Ophthalmology 2001 108 1523-1534.

6 Marcocci C, Bartalena L, Tanda ML, Manetti L, Dell'Unto E, Rocchi R, Barbesino G, Mazzi B, Bartolomei MP, Lepri P, Cartei F, Nardi M \& Pinchera A. Comparison of the effectiveness and tolerability of intravenous or oral glucocorticoids associated with orbital radiotherapy in the management of severe Graves' ophthalmopathy: results of a prospective, single-blind, randomized study. Journal of Clinical Endocrinology and Metabolism 200186 3562-3567.

7 Kahaly GJ, Rosler HP, Pitz S \& Hommel G. Low- versus high-dose radiotherapy for Graves' ophthalmopathy: a randomized, single blind trial. Journal of Clinical Endocrinology and Metabolism $2000 \mathbf{8 5}$ 102-108.

8 Akmansu M, Dirican B, Bora H \& Gurel O. The risk of radiationinduced carcinogenesis after external beam radiotherapy of Graves' orbitopathy. Ophthalmic Research 200335 150-153.

9 Marcocci C, Bartalena L, Rocchi R, Marino M, Menconi F, Morabito E, Mazzi B, Mazzeo S, Sartini MS, Nardi M, Cartei F, Cionini L \& Pinchera A. Long-term safety of orbital radiotherapy for Graves' ophthalmopathy. Journal of Clinical Endocrinology and Metabolism 200388 3561-3566.

10 Robertson DM, Buettner H, Gorman CA, Garrity JA, Fatourechi V, Bahn RS, Petersen IA, Stafford SL, Earle JD, Forbes GS, Kline RW, Bergstralh EJ, Offord KP, Rademacher DM, Stanley NM \& Bartley GB. Retinal microvascular abnormalities in patients treated with external radiation for graves ophthalmopathy. Archives of Ophthalmololgy 2003121 652-657.

11 Wakelkamp IM, Tan H, Saeed P, Schlingemann RO, Verbraak FD, Blank LE, Prummel MF \& Wiersinga WM. Orbital irradiation for Graves' ophthalmopathy: is it safe? A long-term follow-up study. Ophthalmology 2004111 1557-1562.

12 Bartley GB, Fatourechi V, Kadrmas EF, Jacobsen SJ, Ilstrup DM, Garrity JA \& Gorman CA. The incidence of Graves' ophthalmopathy in Olmsted County, Minnesota. American Journal of Ophthalmology 1995120 511-517.

13 Burch HB \& Wartofsky L. Graves' ophthalmopathy: current concepts regarding pathogenesis and management. Endocrine Reviews 199314 747-793.

Received 15 March 2006

Accepted 3 May 2006 